\title{
Asymptomatic Transient Lingual Hyperpigmentation
}

\author{
Nirali Patel, MS; Christina L. Harview, MD; Jennifer L. Hsiao, MD
}

\section{Eligible for 1 MOC SA Credit From the ABD}

This Photo Challenge in our print edition is eligible for 1 self-assessment credit for Maintenance of Certification from the American Board of Dermatology (ABD). After completing this activity, diplomates can visit the ABD website (http://www.abderm.org) to self-report the credits under the activity title "Cutis Photo Challenge." You may report the credit after each activity is completed or after accumulating multiple credits.
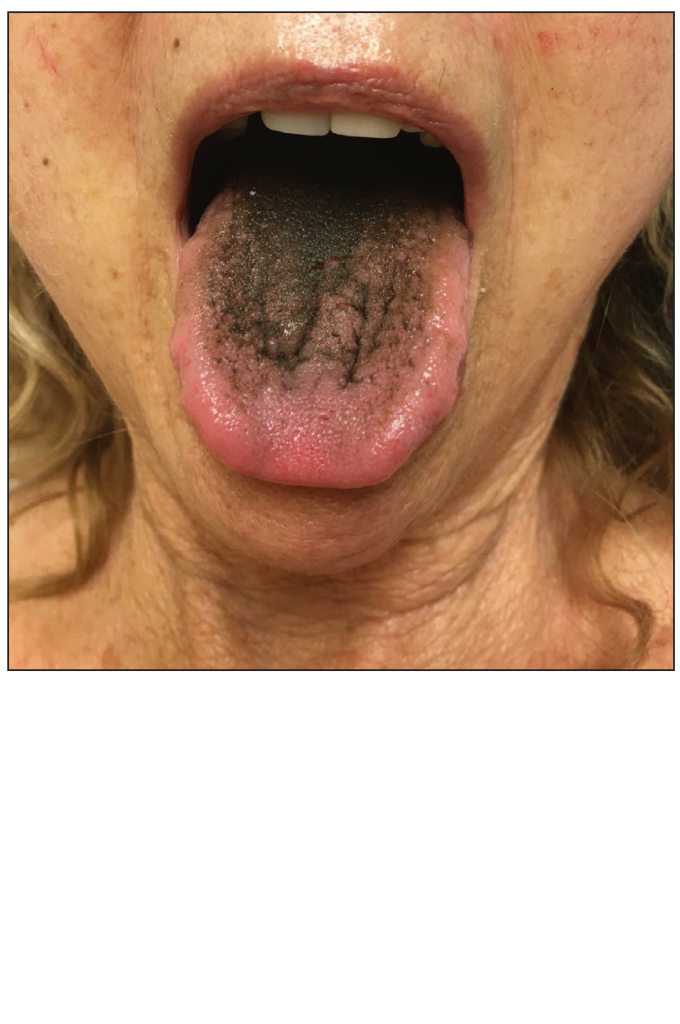

A 77-year-old woman incidentally was noted to have black discoloration of the tongue during a routine dermatologic examination. The patient was unaware of the tongue discoloration and reported that her tongue appeared normal the day prior. The tongue was asymptomatic. Clinical examination revealed black hyperpigmentation on the dorsal aspect of the tongue without appreciable hypertrophy or hyperkeratosis of the filiform papillae. The patient had a half-pack daily smoking habit for many years but had abstained from any smoking or tobacco use for the last 15 years. The patient endorsed good oral hygiene. Upon further questioning, the patient revealed that she had ingested 1 tablet of bismuth salicylate the prior night to relieve postprandial dyspepsia. A cotton-tipped applicator was rubbed gently against the affected area and removed some of the black pigment.

\section{WHAT'S YOUR DIAGNOSIS?}
a. acanthosis nigricans
b. lingua villosa nigra
c. oral hairy leukoplakia
d. pigmented fungiform papillae of the tongue
e. pseudo-black hairy tongue 


\section{THE DIAGNOSIS: Pseudo-Black Hairy Tongue}

P seudo-black hairy tongue is a benign and painless disorder characterized by transient hyperpigmentation of the tongue with a substance that can be easily scraped off. In this case, the patient's lingual discoloration was secondary to the ingestion of bismuth salicylate. The phenomenon is thought to occur due to a reaction between bismuth and sulfur-containing compounds in the saliva, resulting in the characteristic black substance on the surface of the tongue that nestles between the lingual papillae. ${ }^{1}$ An associated feature may include black stools. Other etiologic factors involved in pseudo-black hairy tongue include food coloring, tobacco, and other drugs such antibiotics and antidepressants. ${ }^{2}$

The differential diagnosis of lingual hyperpigmentation includes lingua villosa nigra (also known as black hairy tongue), pigmented fungiform papillae of the tongue, acanthosis nigricans, and oral hairy leukoplakia. Lingua villosa nigra is a similar condition in which individuals present with a black tongue; however, the tongue also appears hairy. The tongue may appear as other colors such as brown, yellow, or green. Patients additionally may have symptoms of burning, dysgeusia, halitosis, or gagging. Poor oral hygiene, xerostomia, use of tobacco or alcohol, and different medications including antibiotics and antipsychotic medications increase the risk for developing lingua villosa nigra. ${ }^{2,3}$ This condition is distinguished from pseudo-black hairy tongue by proliferation and elongation of the filiform papillae. ${ }^{3}$ Pigmented fungiform papillae of the tongue is a normal variant of tongue morphology, is more common in individuals with darker skin types, and primarily affects the lateral aspect and apex of the tongue. ${ }^{4}$ Acanthosis nigricans can appear in the oral cavity as multiple pigmented papillary lesions on the dorsal and lateral regions of the tongue and frequently involves the lips; this condition may be associated with metabolic disorders or underlying malignancy. ${ }^{2,3}$ Oral hairy leukoplakia is caused by Epstein-Barr virus infection and typically presents as white plaques on the dorsal and ventral surfaces of the tongue; this condition largely is found in immunocompromised patients. ${ }^{5}$

In our patient there was an acute onset of tongue discoloration associated with ingestion of bismuth salicylate, no hypertrophy or lengthening of the lingual papillae, and no involvement of the patient's lips, which was consistent with the diagnosis of pseudo-black hairy tongue. Pseudo-black hairy tongue is transient and treated by discontinuation of offending agents and proper hygiene practices.

\section{REFERENCES}

1. Bradley B, Singleton M, Lin Wan Po A. Bismuth toxicity-a reassessment. J Clin Pharm Ther. 1989;14:423-441.

2. Gurvits GE, Tan A. Black hairy tongue syndrome. World J Gastroenterol. 2014;20:10845-10850.

3. Schlager E, St Claire C, Ashack K, et al. Black hairy tongue: predisposing factors, diagnosis, and treatment. Am J Clin Dermatol. 2017;18:563-569.

4. Mangold AR, Torgerson RR, Rogers RS. Diseases of the tongue. Clin Dermatol. 2016;34:458-469.

5. Husak R, Garbe C, Orfanos CE. Oral hairy leukoplakia in 71 HIVseropositive patients: clinical symptoms, relation to immunologic status, and prognostic significance. J Am Acad Dermatol. 1996;35:928-934. 